\title{
Surface Instability of Microscale Multilayer Thin Film System
}

Yueguang Wei, Di Jiang, Ajing Cao, Haifeng Zhao

LNM, Institute of Mechanics, Chinese Academy of Sciences, Beijing 100080, P.R. China

\begin{abstract}
Fundamental relations of surface instability for micro-scale multilayer thin film systems are derived based on the Hill and Hutchinson bifurcation theory and the volume average integral. In the present relations, a size effect is considered through generalizing the plastic hardening modulus to include the strain gradient hardening effects. By using the model, firstly, the parameter-controlled instability region is divided and analyzed, secondly, the surface instability of the multilayer thin films are analyzed. In the surface instability analysis, several kinds of failure band formations are obtained, such as the kink bands and the compressive buckling bands in vertical direction and in inclined direction. Moreover, the failure mechanism of the multilayer thin film system due to surface instability is used to interpret the microstructure features of the surface-nanocrystallization materials.
\end{abstract}

\section{INTRODUCTION}

The micro-scale multilayer thin film system (MMTFS), with its special characteristics, is relevant to many advanced materials applications and widely used in the MEMS- as well as surface- and interfacial-engineering areas. Fundamental roles in the MEMS and in the advanced materials played by the MMTFS can be described as: protecting, connecting, strengthening, toughening, as well as some function devices, etc. [1,2]. Typical failure modes of MMTFS include interfacial cracking, thin film cracking perpendicular to the interface, and failure initiating at the surface, etc. The last failure formation is caused by material surface instability. It is well known that due to the residual compressive stress related to film deposition, or direct external compression, surface instability occurs as the main failure mode. In this case, the surface kink bands and damage bands are formed near the material surface. Additionally, the surface instability is accompanied by some kinds of failure patterns appearing on the material surface, such as surface layer thin film delamination, buckling, damaging, etc. The surface instability is as a major failure formation not only for a multilayer thin film material, but also for a uniform or a single crystal material, such as the damage bands within the surface layer of nanocrystalline Al [3], as shown in Figures 1(a) and (b).

In order to investigate the failure mechanism related to surface instability, in the present research, a theoretical model considering strain gradient effect is presented and developed based on the Hill and Hill-Hutchinson's bifurcation theory [4-6]. The surface instability description for the MMTFS is formulated through homogenizing a set of field equations of multilayer uniform thin film materials. Motivated by the development of strain gradient hardening theory, the surface instability analysis is extended to the micro-scale case through generalizing the plastic hardening modulus to include the strain gradient effect. The surface failure patterns, such as the kink bands, etc., are modeled and computed through bifurcation and post-bifurcation analyses.

\section{ELASTIC-PLASTIC BIFURCATION THEORY OF UNIFORM MATERIALS}

Firstly, let's recall the bifurcation theory. Elastic-plastic fundamental equations of bifurcation for uniform materials can be written as follows [4] 


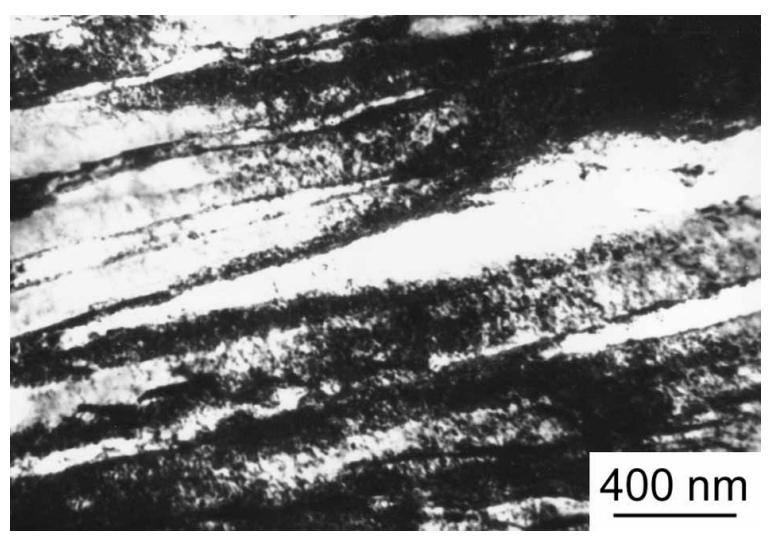

(a)

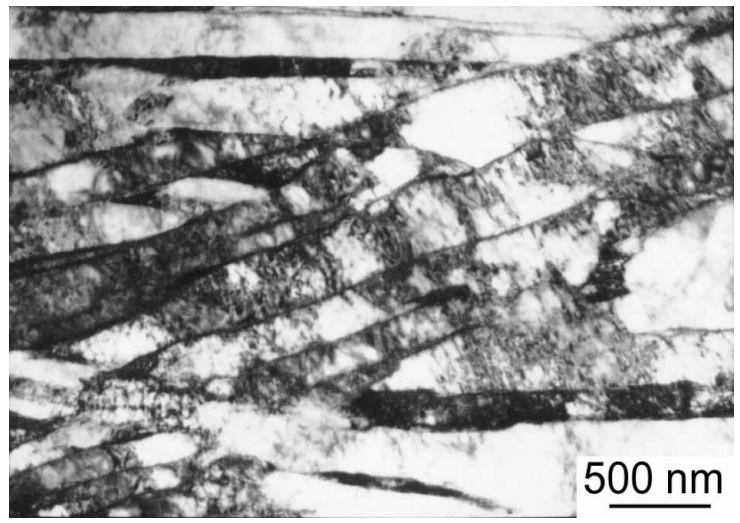

(b)

Figure 1. TEM micrographs showing the formed microbands within surface layer due to surface-nanocrystallization [3].

$$
\left\{\begin{array}{l}
\dot{t}_{i j, i}=0 \\
D_{i j}=\frac{1}{2}\left(v_{i, j}+v_{j, i}\right) \\
\dot{t}_{i j}=\sigma_{i k} v_{j, k}-\left(\sigma_{i k} D_{j k}+\sigma_{j k} D_{i k}\right)+K_{i j k l}\left(D_{k l}-D_{k l}^{P}\right)
\end{array}\right.
$$

where $\dot{t}_{i j}$ is the normal stress rate, $v_{i}$ is velocity, $D_{i j}$ is the velocity gradients, $\sigma_{i j}$ is the Cauchy stress, $K_{i j k l}$ is elastic modulus,

$$
K_{i j k l}=\lambda \delta_{i j} \delta_{k l}+\mu\left(\delta_{i k} \delta_{j l}+\delta_{i l} \delta_{j k}\right)
$$

$(i, j, k, l=1,3), D^{P}{ }_{i j}$ is the plastic tensor of the velocity gradients, and can be expressed as follows,

$$
D_{k l}^{P}= \begin{cases}\frac{Q_{i j} K_{i j m n} D_{m n}}{Q_{q r} K_{q r s t} Q_{s t}+H} Q_{k l} & Q_{i j} K_{i j k l} D_{k l} \geq 0 \\ 0 & Q_{i j} K_{i j k l} D_{k l}<0\end{cases}
$$

Where $Q_{i j}$ is unit external normal tensor in stress space, which can be expressed as

$$
Q_{i j}=\sigma_{i j}^{\prime} / \sqrt{\sigma_{k l}^{\prime} \sigma_{k l}^{\prime}}
$$

$\sigma_{i j}^{\prime}=\sigma_{i j}-\frac{1}{3} \sigma_{k k} \delta_{i j}$ is stress devitoric, $H$ in (3) is plastic hardening modulus, can be determined from experiment through measuring material stress strain curve, $H=\sigma / \varepsilon^{P}=g\left(\varepsilon^{P}, \eta\right)$, where $\left(\sigma, \varepsilon^{P}, \eta\right)$ are flow stress $\left(\sigma=\sqrt{\sigma_{e}^{2}+l \eta}, \sigma_{e}\right.$ is effective stress [7]), effective plastic strain and effective strain gradient, respectively. $g\left(\varepsilon^{P}, \eta\right)$ can be determined in experiment through measuring the macro-scale stress-strain relation $\left(\sigma_{e} \sim \varepsilon_{e}\right)$, where $\varepsilon_{e}$ is effective strain, and $\varepsilon_{e}-\sigma_{e} / E=\varepsilon^{P} . H$ has been considered to include the strain gradient effect [7-10]. Therefore, present analyses are suitable for micro-scale case. 
For the incompressible plane strain elastic-plastic case, fundamental equations can be expressed as follows

$$
\left\{\begin{array}{l}
\dot{t}_{11,1}+\dot{t}_{21,2}=0, \quad \dot{t}_{12,1}+\dot{t}_{22,2}=0, \\
v_{1,1}+v_{2,2}=0, \\
\dot{t}_{11}-\dot{t}_{22}=2\left(2 \mu \mu^{*}-\frac{1}{2} \sigma\right) v_{1,1}, \\
\dot{t}_{12}=\left(\mu+\frac{1}{2} \sigma\right) v_{2,1}+\left(\mu-\frac{1}{2} \sigma\right) v_{1,2}, \\
\dot{t}_{21}=\left(\mu-\frac{1}{2} \sigma\right)\left(v_{2,1}+v_{1,2}\right)
\end{array}\right.
$$

where $\quad \mu^{*}=\frac{\mu H}{2 \mu+H}$

Eq. (5) is the fundamental relation of instability for a uniform material in plane strain case. In order to look for a characteristic solution of instability for Eq. (5), a velocity potential function $\varphi\left(x_{1}, x_{2}\right)$ is introduced, and is defined as

$$
v_{1}=\frac{\partial \varphi}{\partial x_{2}}, \quad v_{2}=-\frac{\partial \varphi}{\partial x_{1}}
$$

So, finding the characteristic solution of Eq. (5) is equivalent to solving the characteristic differential equation

$$
\left(\mu+\frac{1}{2} \sigma\right) \frac{\partial^{4} \varphi}{\partial x_{1}^{4}}+2\left(2 \mu^{*}-\mu\right) \frac{\partial^{4} \varphi}{\partial x_{1}^{2} \partial x_{2}^{2}}+\left(\mu-\frac{1}{2} \sigma\right) \frac{\partial^{4} \varphi}{\partial x_{2}^{4}}=0
$$

Through considering a characteristic solution form of

$$
\varphi=\varphi\left(k x_{1}+x_{2}\right)
$$

and through determining the types of the equation (8) corresponding characteristic value of $k$, the instability features can be discussed. Substituting (9) to (8), one can arrive at a characteristic parameter equation

$$
\left(\mu+\frac{1}{2} \sigma\right) k^{4}+2\left(2 \mu^{*}-\mu\right) k^{2}+\left(\mu-\frac{1}{2} \sigma\right)=0
$$

Types of equation (8), elliptical type $(\mathrm{E})$, hyperbolic type $(\mathrm{H})$ or parabolic type $(\mathrm{P})$, depend on the characteristics of roots of $k$ in (10):

$$
\begin{aligned}
& \frac{|\sigma|}{4 \mu^{*}} \leq \frac{\mu}{2 \mu^{*}} \leq 1, \quad \text { or } 1+\left(\frac{\sigma}{4 \mu^{*}}\right)^{2} \leq \frac{\mu}{2 \mu^{*}} \\
& 1 \leq \frac{|\sigma|}{4 \mu^{*}} \leq \frac{\mu}{2 \mu^{*}} \leq 1+\left(\frac{\sigma}{4 \mu^{*}}\right)^{2} \\
& \mu \leq \frac{1}{2}|\sigma|
\end{aligned}
$$




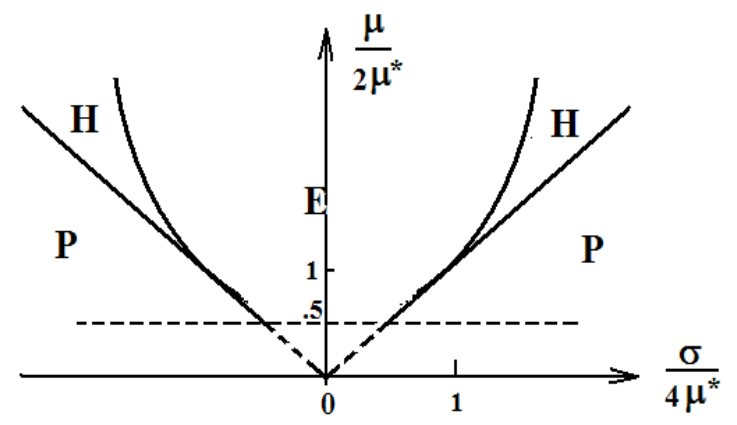

Figure 2. Parameter regions of bifurcations.

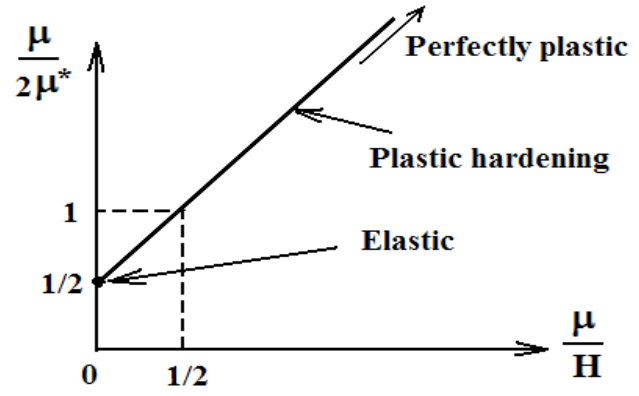

Figure 3. Plastic hardening modulus $(\mathrm{H})$.

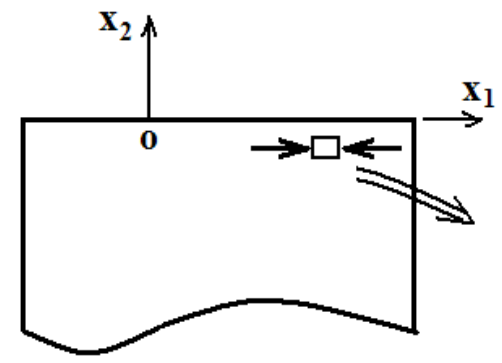

(a)

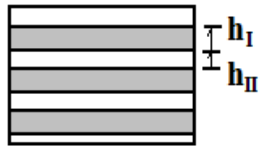

(b)

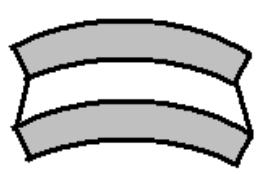

(c)

Figure 4. Homogenizing cell model of multilayer thin film system.

The corresponding parameter regions of the Eqs. (11)-(13) are plotted in Figure 2. On the other hand, from Eq. (6), one can plot the combination parameter relationship of $\mu / 2 \mu^{*} \sim \mu / H$ (Figure 3). From Figure 3, the combination parameter $\mu / 2 \mu^{*}$ should be larger than $1 / 2$ (see dashed line in Figure 2).

\section{ELASTIC-PLASTIC BIFURCATION OF MULTILAYER THIN FILM SYSTEM}

Let's consider the case of the multilayer thin films with two-layer periodic microstructure. During instability, each material deformation satisfies the equations (5). Consider a microscale cell model, as shown in Figure 4. Through volume average integration over the cell volume (referring to [11,12] for the fiber-reinforced composite), one can obtain the bifurcation equations as

$$
\left\{\begin{array}{l}
\dot{t}_{11,1}+\dot{t}_{21,2}=0, \quad \dot{t}_{12,1}+\dot{t}_{22,2}=0, \\
v_{1,1}+v_{2,2}=0, \\
\dot{t}_{11}-\dot{t}_{22}=2\left(2 \mu *-\frac{1}{2} \sigma\right) v_{1,1}, \\
\dot{t}_{21}=\left(V_{I} / b_{I}+V_{I I} / b_{I I}\right)^{-1}\left(v_{2,1}+v_{1,2}\right), \\
\dot{t}_{12}=\left(V_{I} / b_{I}+V_{I I} / b_{I I}\right)^{-1}\left[\left(V_{I} a_{I} / b_{I}+V_{I I} a_{I I} / b_{I I}\right)\right. \\
\left.+V_{I} V_{I I}\left(a_{I}-b_{I}-a_{I I}\right)\left(b_{I I}^{-1}-b_{I}^{-1}\right)\right] v_{2,1}+\left(V_{I} / b_{I}+V_{I I} / b_{I I}\right)^{-1} v_{1,2}
\end{array}\right.
$$

where 


$$
\begin{aligned}
& \mu^{*}=V_{I} \mu_{I}^{*}+V_{I I} \mu_{I I}^{*}, \sigma=V_{I} \sigma_{I}+V_{I I} \sigma_{I I}, \dot{t}_{1 i}=V_{I} \dot{t}_{1 i I}+V_{I I} \dot{t}_{1 i I I}, \\
& \dot{t}_{2 i I}=\dot{t}_{2 i I I}=\dot{t}_{2 i}, \quad v_{i I}=v_{i I I}=v_{i}, a_{\gamma}=\left(\mu_{\gamma}+\frac{1}{2} \sigma_{\gamma}\right), b_{\gamma}=\left(\mu_{\gamma}-\frac{1}{2} \sigma_{\gamma}\right) . \\
& (i=1,2 ; \gamma=I, I I)
\end{aligned}
$$

$V_{I}$ and $V_{I I}$ are volume fractions of two-layer periodic microstructures, respectively, $V_{I}+V_{I I}=1$. If $b_{I I} / b_{I}$ and $\sigma_{I} / \mu_{I}$ are small quantities and neglected, Eq. (14) will be reduced to the form of Eq. (5), however, corresponding shear modulus will be replaced by,

$$
\mu=\frac{\mu_{I I}}{V_{I I}}-\frac{\sigma_{I I}}{2 V_{I I}}+\frac{\sigma}{2}
$$

where $\sigma$ expression is given in Eq. (15). From Eq. (16), when the MMTFS with the two-layer periodic microstructure is homogenized into an equivalent material, the equivalent shear modulus is a function of external loads.

\section{SURFACE INSTABILITY OF MULTILAYER THIN FILMS}

For surface instability of multilayer thin films, let's discuss a specific form of velocity potential function (Eq. (9)) as follows

$$
\varphi=f\left(x_{2}\right) \cos c x_{1}, \quad c=m \pi / \lambda . \quad(m=1,2, \ldots)
$$

where $\lambda$ is wavelength of the instability model along $x_{1}$ direction.

\section{Vertical instability bands}

Substituting (17) into (8) and considering the first formula of (11) and free surface boundary condition, one can obtain solution

$$
\begin{gathered}
\varphi=A\left[e^{c q_{1} x_{2}}-\left(1+q_{1}^{2}\right)\left(1+q_{2}^{2}\right)^{-1} e^{c q_{2} x_{2}}\right] \cos c x_{1} \\
q_{1,2}=\left\{\left[\left(2 \mu^{*}-\mu\right) \pm \sqrt{\left(2 \mu^{*}-\mu\right)^{2}-\left(\mu^{2}-\frac{1}{4} \sigma^{2}\right)}\right] /\left(\mu-\frac{1}{2} \sigma\right)\right\}^{1 / 2} \\
\frac{2 \mu^{*}}{\mu}=\frac{|\sigma|}{2 \mu}\left[\sqrt{\left(1+\frac{|\sigma|}{2 \mu}\right) /\left(1-\frac{|\sigma|}{2 \mu}\right)}-1\right] .
\end{gathered}
$$

\section{Inclined instability bands}

Substituting (17) into (8) and considering the second formula of (11) and free surface boundary condition, one can obtain solution

$$
\begin{gathered}
\varphi=\frac{1}{2} A e^{c q x_{2}}\left\{\begin{array}{l}
\cos c\left(p x_{2}+x_{1}\right)+\cos c\left(p x_{2}-x_{1}\right) \\
-\frac{1+q^{2}-p^{2}}{2 p q}\left[\sin c\left(p x_{2}+x_{1}\right)+\sin c\left(p x_{2}-x_{1}\right)\right]
\end{array}\right\} \\
p, q=\left[ \pm\left(\mu-2 \mu^{*}\right) /(2 \mu-\sigma)+\frac{1}{2} \sqrt{(2 \mu+\sigma) /(2 \mu-\sigma)}\right]^{1 / 2} \\
\frac{2 \mu^{*}}{\mu}=\frac{|\sigma|}{2 \mu}\left[\sqrt{\left(1+\frac{|\sigma|}{2 \mu}\right) /\left(1-\frac{|\sigma|}{2 \mu}\right)}-1\right] .
\end{gathered}
$$


From solutions given in Eqs. (18) and (19), one can obtain the failure bands, as shown in Figure 5, where (a) and (b) are compressive buckling bands, (c) and (d) are kink bands, (a) and (c) are vertical instability bands, and (b) and (d) are inclined instability bands.

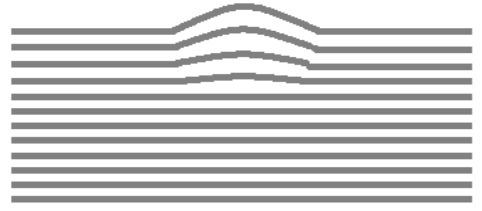

(a)

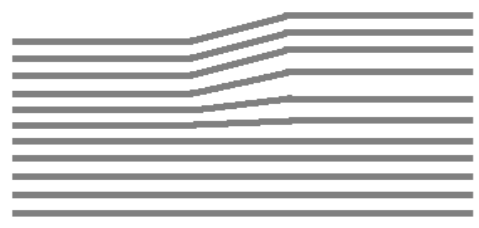

(c)

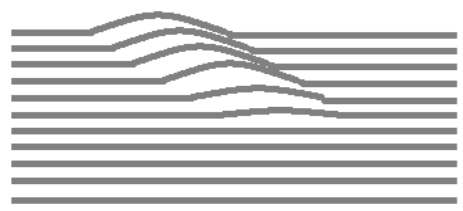

(b)

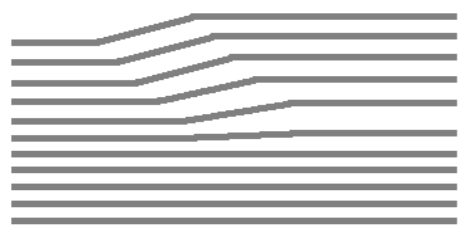

(d)

Figure 5. The compressive buckling bands ( $a, b)$ and kink bands (c, d) are formed due to surface instability. (a, c) are vertical failure bands, and (b, d) are inclined failure bands.

\section{CONCLUSIONS}

The surface instability of the MMTFS has been investigated based on the bifurcation theory of Hill and Hutchinson. Through generalizing the plastic hardening modulus to include the strain gradient effect, the analysis can be used to the micro-scale bifurcation. In the surface instability, the kink bands or compressive buckling bands along vertical or inclined directions are formed near the material surface. As the instability bands nucleates and propagates, the band transection cracking within the band is taken place. From this point, the microstructure features of the surface-nanocrystalline materials can be interpreted.

\section{ACKNOWLEDGEMENTS}

This work was supported by the National Natural Science Foundation of China through Grants Nos. 10432050 and 10428207.

\section{REFERENCES}

1. R. P. Vinci, S. P. Baker, MRS Bulletin 27, 52(2002).

2. M. Yamamoto, Y. Hotta, M. Sato, Thin solid films 433, 224(2003).

3. Y. Wei, X. Wu, C. Zhu, M. Zhao, J. Mater. Res., (2004). (Submitted)

4. R. Hill, J. Mech. Phys. Solids 10, 1(1962).

5. R. Hill, J. W. Hutchinson, J. Mech. Phys. Solids 23, 238(1975).

6. N. J. B. Young, J. Mech. Phys. Solids 24, 77(1976).

7. W. D. Nix, H. Gao, J Mech Phys Solids 46, 411(1998).

8. N. A. Fleck, J. W. Hutchinson, Adv. in Appl. Mech. 33, 295(1997).

9. H. Gao, Y. Huang, W. D. Nix, J. W. Hutchinson, J Mech Phys Solids 47, 1239 (1999).

10. Y. Wei, X. Wang, X. Wu, Y. Bai, Science in China (Series A) 44, 74 (2001).

11. W. Yang, Y. Wei, Int. J. Damage Mech. 1, 80(1992).

12. Y. Wei, W. Yang, K. C. Hwang, Science in China (Series A) 37, 1077(1994). 